\title{
Piezoelectric and Semiconducting Coupled Power Generating Process of a Single ZnO Belt/Wire. A Technology for Harvesting Electricity from the Environment
}

2006

Vol. 6, No. 8

$1656-1662$

\author{
Jinhui Song, Jun Zhou, and Zhong Lin Wang* \\ School of Materials Science and Engineering, Georgia Institute of Technology, \\ Atlanta, Georgia 30332-0245
}

Received April 11, 2006; Revised Manuscript Received July 6, 2006

\begin{abstract}
This paper presents the experimental observation of piezoelectric generation from a single $\mathrm{ZnO}$ wire/belt for illustrating a fundamental process of converting mechanical energy into electricity at nanoscale. By deflecting a wire/belt using a conductive atomic force microscope tip in contact mode, the energy is first created by the deflection force and stored by piezoelectric potential, and later converts into piezoelectric energy. The mechanism of the generator is a result of coupled semiconducting and piezoelectric properties of $\mathrm{ZnO}$. A piezoelectric effect is required to create electric potential of ionic charges from elastic deformation; semiconducting property is necessary to separate and maintain the charges and then release the potential via the rectifying behavior of the Schottky barrier at the metal-ZnO interface, which serves as a switch in the entire process. The good conductivity of $\mathrm{ZnO}$ is rather unique because it makes the current flow possible. This paper demonstrates a principle for harvesting energy from the environment. The technology has the potential of converting mechanical movement energy (such as body movement, muscle stretching, blood pressure), vibration energy (such as acoustic/ultrasonic wave), and hydraulic energy (such as flow of body fluid, blood flow, contraction of blood vessels) into electric energy that may be sufficient for self-powering nanodevices and nanosystems in applications such as in situ, real-time, and implantable biosensing, biomedical monitoring, and biodetection.
\end{abstract}

Among the known one-dimensional nanomaterials, zinc oxide $(\mathrm{ZnO})$ is unique because of its semiconducting and piezoelectric dual properties. ${ }^{1-4}$ Most of the applications of $\mathrm{ZnO}$ utilize either the semiconducting ${ }^{5}$ or piezoelectric property, ${ }^{6,7}$ and there are few cases where the coupled semiconducting-piezoelectric dual properties are fully employed. Recently, we have demonstrated an innovative application of aligned $\mathrm{ZnO}$ nanowire arrays for converting nanoscale mechanical energy into electric energy. ${ }^{8}$ The operation mechanism of the electric generator relies on the unique coupling of piezoelectric and semiconducting dual properties of $\mathrm{ZnO}$ as well as the elegant rectifying function of the Schottky barrier formed between the metal tip and the nanowire. ${ }^{9}$ The approach has the potential of converting biological mechanical energy, acoustic/ultrasonic vibration energy, and biofluid hydraulic energy, into electricity, demonstrating a new path way for self-powering of wireless nanodevices and nanosystems. This research opens a new chapter in nanowire (NW) and nanobelt (NB) applications, especially for the

\footnotetext{
* To whom correspondence should be addressed. E-mail: zhong.wang@mse.gatech.edu.
}

wurtzite structured materials, such as $\mathrm{ZnO}, \mathrm{GaN}$, and $\mathrm{ZnS}$, and it demonstrates the prosperity of self-powering nanodevices and nanosystems built using NWs and NBs for optoelectronics, ${ }^{10}$ biosensors, ${ }^{11}$ resonators, ${ }^{12}$ and more.

Our original work was based on measurements on an array of aligned $\mathrm{ZnO}$ nanowires. In this paper, the detailed piezoelectric discharging process of a single $\mathrm{ZnO}$ wire/belt is examined. By directly correlating the manipulation process of a wire/belt and the electric output voltage/current signal under atomic force microscopy, the details about the generation mechanism have been revealed. Discussion is given about the potential applications of this energy harvesting process from the environment.

Our experiment was based on mechanical manipulation of a single $\mathrm{ZnO}$ wire/belt by an atomic force microscope. By use of a long $\mathrm{ZnO}$ wire/belt that was large enough to be seen under an optical microscope, one end of the $\mathrm{ZnO}$ wire was affixed on a silicon substrate by silver paste, while the other end was left free. The substrate was an intrinsic silicon, so its conductivity was rather poor. The wire laid on the substrate but kept a small distance from the substrate to eliminate the friction with the substrate except at the affixed 

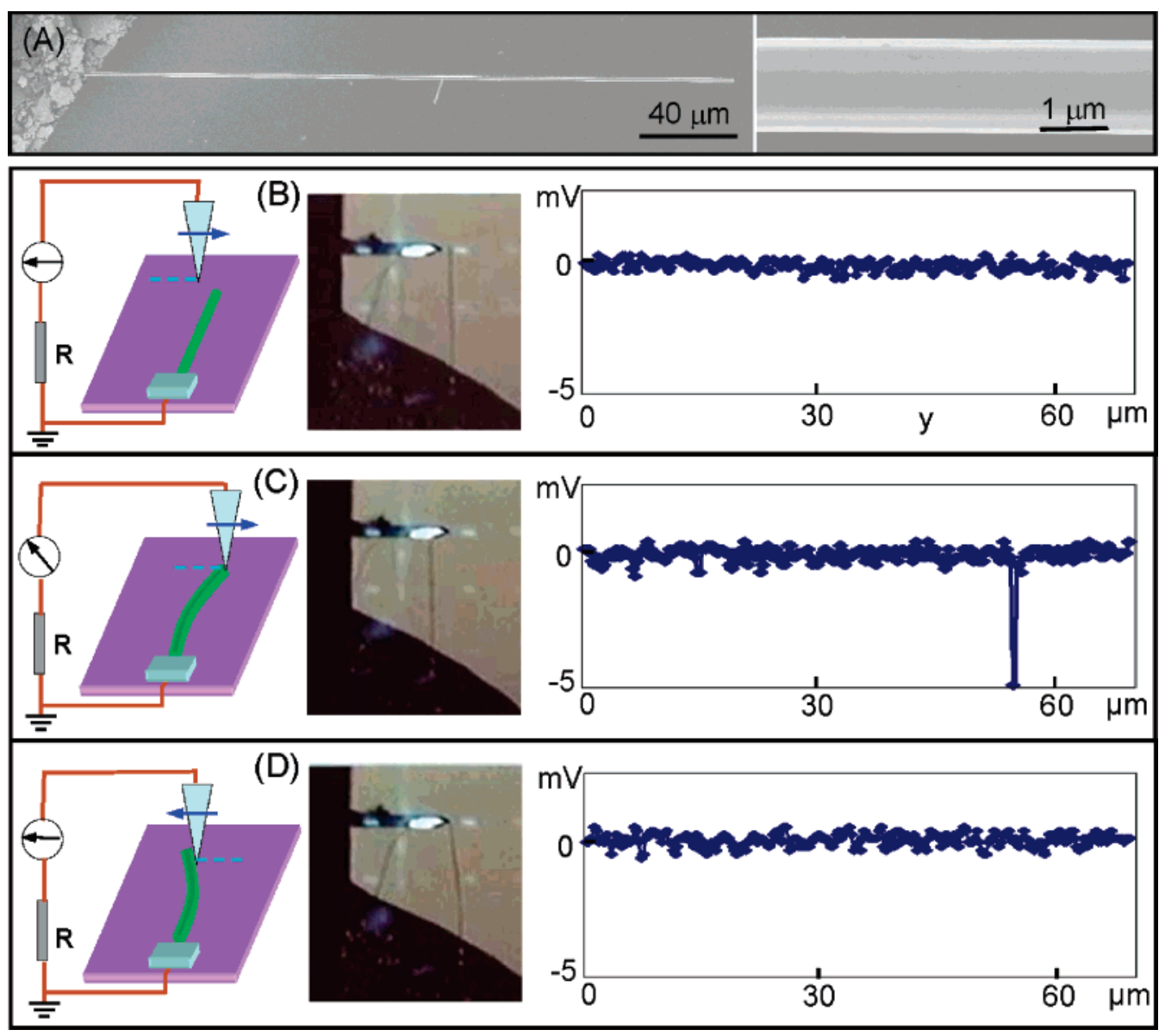

Figure 1. In situ observation of the process for converting mechanical energy into electric energy by a piezoelectric ZnO wire. (A) SEM images of a $\mathrm{ZnO}$ wire with one end affixed by silver paste onto a silicon substrate and the other end free. The wire has a hexagonal cross section. (B, C, and D) Three characteristic snapshots and the corresponding output voltage images when the tip scanned across the wire. The schematic illustration of the experimental condition is shown at the left-hand side, with the scanning direction of the tip indicated by an arrowhead. The scanning speed of the AFM tip was $105.57 \mu \mathrm{m} / \mathrm{s}$, and the sampling density is 256 per line. (B) The AFM tip scanned over the top of the wire on the silicon substrate, and the voltage output is just in the noise level. (C) The AFM tip touched the top end of the wire, and the output voltage image showed a sharp negative peak. (D) Right after the AFM tip passed the top end of the wire and scanned toward its lower part, but without across the wire. No output voltage was detected.

side (Figure 1A). The measurements were performed by atomic force microscopy (AFM) (Molecular Force Probe MFP-3D from Asylum Research) using a Si tip coated with Pt film (Electri-Lever AC240 from Olympus), which had a tetrahedral shape with an apex angle of $70^{\circ}$ and height of $14 \mu \mathrm{m}$ and a spring constant of $1.42 \mathrm{~N} / \mathrm{m}$. The measurements were done in AFM contact mode under a constant normal force of $5 \mathrm{nN}$ between the tip and sample surface, and the scan area was $70 \times 70 \mu \mathrm{m}^{2}$.

Both the topography (feedback signal from the scanner) and the corresponding output voltage $(V)$ images across a load were recorded simultaneously when the AFM tip was scanned across a wire/belt. The topography image reflects the change in normal force perpendicular to the substrate, which shows a bump only when the tip scans over the wire. The output voltage between the conductive tip and the ground was continuously monitored as the tip scans over the wire/ belt. No external voltage was applied in any stage of the experiment. The entire experimental process and the output images were captured by video recording (see Supporting Information), so that we were able to directly visualize the electric generation process. The output data to be presented were obtained from snapshots of the characteristic events observed during the experiments.

The AFM tip scanned line-by-line at a speed of 105.57 $\mu \mathrm{m} / \mathrm{s}$ perpendicular to the wire either from above the top end to the lower part of the wire or from the lower part toward the top end. For a wire with hexagonal cross section (Figure 1A), three characteristics features were observed. When the tip scanned above the top end of the wire without touching the wire but the silicon substrate, the output voltage signal was nothing but noise (Figure 1B). When the tip scanned until it touched the top end of the wire, a spark output voltage signal was observed (Figure 1C). The output voltage is negative for the load $R_{\mathrm{L}}$ for almost all of the observed cases, indicating the tip has a lower potential than the grounded silver paste. When the tip scanned down along the wire, it deflected the wire but could not go over it, and the output voltage showed no peak but noise (Figure 1D).

The observation for a large belt is given in Figure 2. The SEM images clearly display the rectangular cross section of the belt (Figure 2A). In this experiment, we selected a strong 

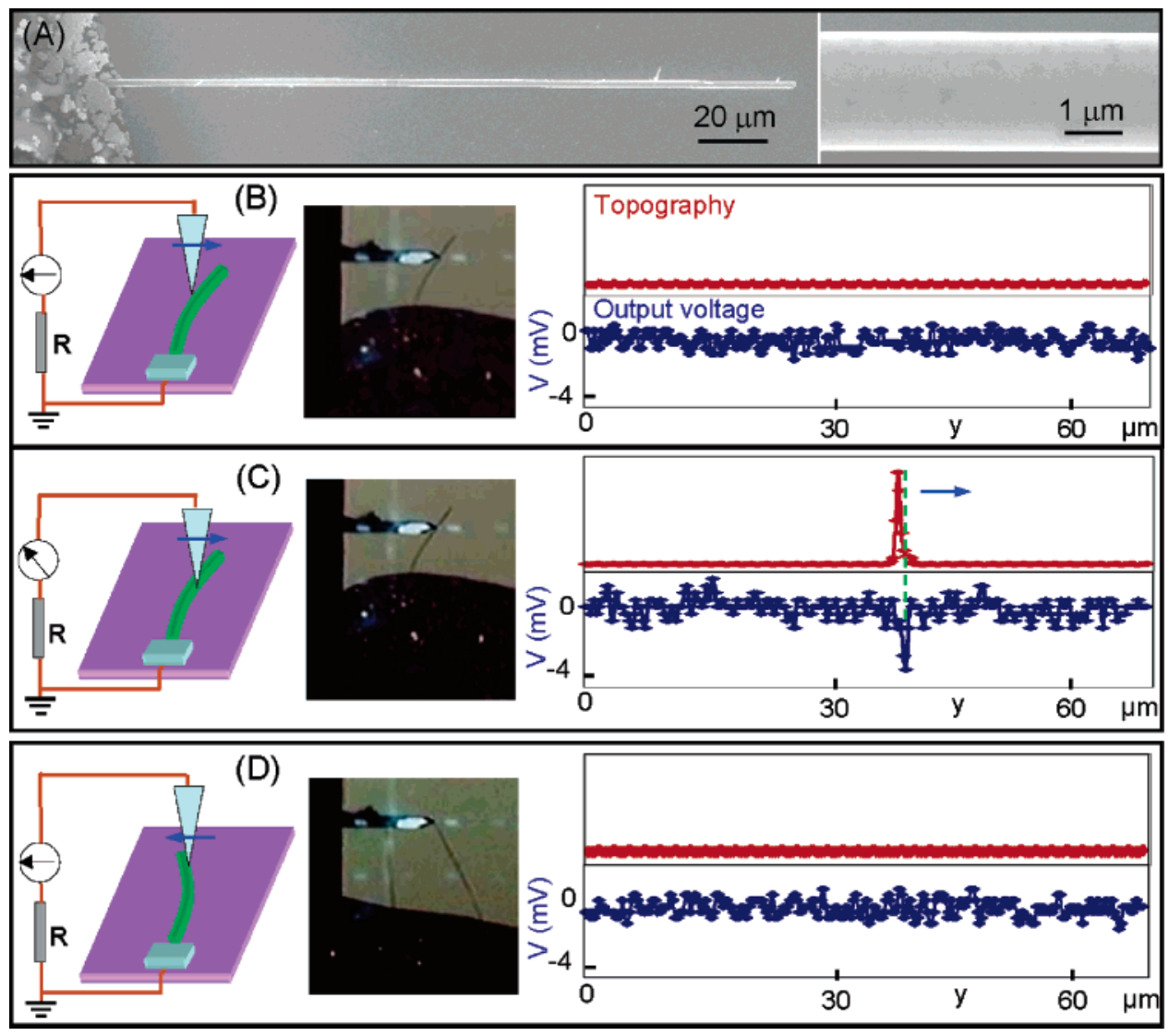

Figure 2. In situ observation of the process for converting mechanical energy into electric energy by a piezoelectric ZnO belt. (A) SEM images of a $\mathrm{ZnO}$ belt with one end affixed by silver paste onto a silicon substrate and the other end free. The belt has a rectangular cross section. (B, C, and D) Three characteristic snapshots and the corresponding topography (red curve) and output voltage (blue curve) images when the tip scanned across the middle section of the belt. The schematic illustration of the experimental condition is shown at the lefthand side, with the scanning direction of the tip indicated by an arrowhead. The scanning speed of the AFM tip was $105.57 \mu \mathrm{m} / \mathrm{s}$, and the sampling density is 256 per line. (B) The AFM tip pushed the belt toward the right-hand side but did not go above and across its width, as judged from the topography image. No output voltage was detected. (C) The AFM tip pushed the belt toward the right-hand side and went above and across its width, as judged from the peak in the topography image. The output voltage image showed a sharp negative peak. There is a delay in the output voltage peak in reference to the normal force image (the peak in the topography image). (D) For an alternative belt, the AFM tip pushes the belt toward the left but did not go above and across its width, as judged from the topography image. No output voltage was detected beyond noise level although the deflection was large.

belt and used an AFM tip to push perpendicular to the belt at its middle section. When subjected to a displacement force, one side of the belt is stretched (left-hand side) and the other side (the right-hand side) is compressed. The objective of this experiment is to observe if the piezoelectric discharge occurs when the tip touches either the stretched side or the compressed side, or both sides. The topography image directly captured if the tip passed over the belt or not because it was a representation of the normal height received by the cantilever. When the tip pushed the wire but did not go over and across it, as judged by the flat output signal in the topography image (Figure 2B), no voltage output was produced, indicating the stretched side produced no piezoelectric discharge event. Once the tip went over the belt and in touch with the compressed side, as indicated by a peak in the topography image, a sharp voltage output peak is observed (Figure 2C). By analyzing the positions of the peaks observed in the topography image and the output voltage image, we noticed that the discharge occurred after the tip nearly finishing across the wire. This clearly indicates that the compressed side was responsible for producing the negative piezoelectric discharge voltage. For another belt, when the tip retracted from the right-hand side to the lefthand side (Figure 2D), no output voltage was detected because the tip just touched the stretched side of the belt without crossing it.

As a summary of the above experimental observations, there are three key results. First, piezoelectric discharge is observed for both wire and belt, and it may occur only when the AFM tip touches the end of the bent wire/belt. Second, the piezoelectric discharge occurs only when the AFM tip touches the compressed side of the wire/belt, and there is no voltage output if the tip touches the stretched side of the wire/belt. Third, the piezoelectric discharge gives negative output voltage as measured from the load $R_{\mathrm{L}}$. Finally, in reference to the topography image, the voltage output event occurs when the AFM tip is about finished crossing the width of the wire/belt, which means that the discharge event is delayed to the last half of the contact between the tip and the wire/belt. 


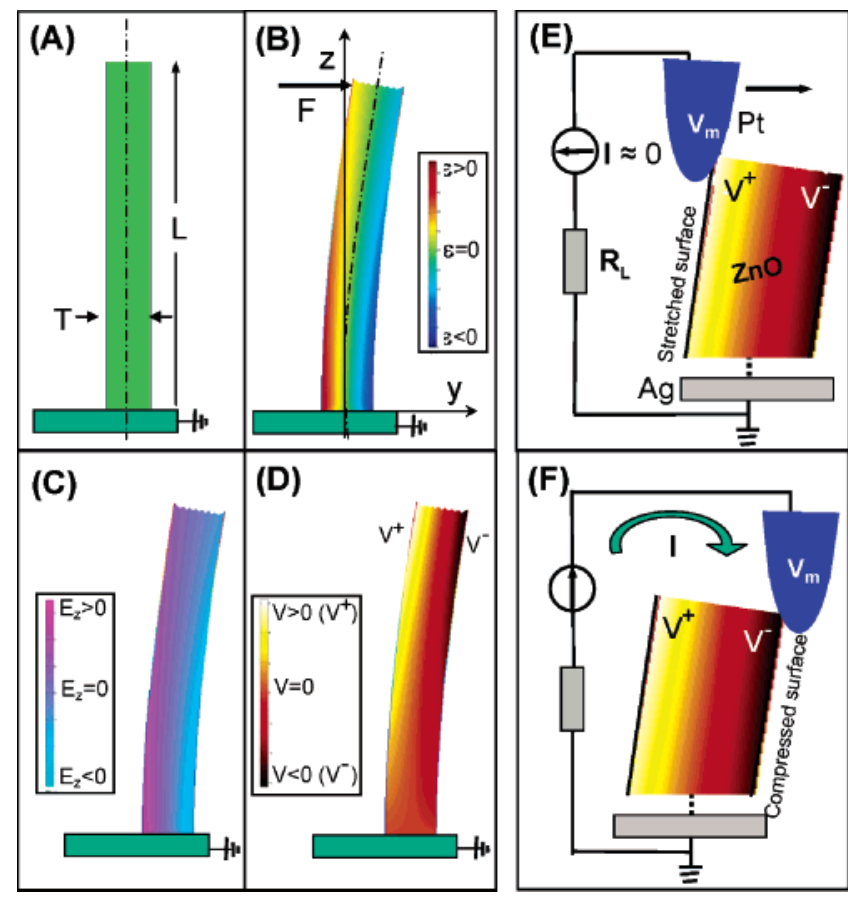

Figure 3. Working mechanism for the power generation process of a piezoelectric $\mathrm{ZnO}$ wire/belt as a result of coupled piezoelectric and semiconducting properties in conjunction with the Schottky barrier at the AFM tip-semiconductor interface. (A) Schematic definition of a belt. (B) Longitudinal strain $\epsilon_{z}$ distribution in the belt after being deflected by an AFM tip from the side. (C) The corresponding longitudinal piezoelectric induced electric field $E_{z}$ distribution in the belt with. (D) Potential distribution in the belt as a result of piezoelectric effect, with the stretched and compressed side surfaces being positive and negative potentials, respectively. (E, F) Metal and semiconductor contacts between the AFM tip and the semiconductor $\mathrm{ZnO}$ belt at two reversed local contact potentials (positive and negative), showing reverse and forward biased Schottky rectifying behavior, respectively. It is this oppositely biased Schottky barrier at the tip-belt interface that makes it possible to preserve the piezoelectric charges and later produce the discharge output. The one-way switching role played by the Schottky diode is important for our observation of the piezoelectric output. The process in (E) is to build up and preserve the charges/potential; the process in $(\mathrm{F})$ is to discharge the potential through a flow of electrons from the circuit. The negative output voltage on the load is a result of current flow from the AFM tip through the $\mathrm{ZnO}$ belt to the ground.

To explain the observed phenomena, we now examine the potential distribution in the wire/belt based on piezoelectric induced potential distribution. Our discussion is for illustrating the physics process and principle rather than a rigorous numerical calculation. We simply consider the polarization introduced in a belt as a result of elastic deformation. ${ }^{13}$ The relationship between strain $(\epsilon)$ and the local piezoelectric field $(E)$ is given by $\epsilon=d E$, where $d$ is the piezoelectric coefficient. For a belt of thickness $T$ and length $L$ as shown in Figure 3A, under the displacement of an external force $F$ from the AFM tip applied perpendicularly at the top of the belt $(z=L)$, a strain field in the belt would be formed. For any segment of the belt along its length, the local bending is described by its local curvature $1 / R$, where $R$ is the local radius for describing the bending of the belt, which is related to the shape of the belt by

$$
\frac{1}{R}=\frac{\mathrm{d}^{2} y}{\mathrm{~d} z^{2}}
$$

which was from the geometrical shape of the curved belt as described in calculus (see Figure 3B). The shape of the belt can be described by the static deflection equation of the belt

$$
\frac{\mathrm{d}^{2} y}{\mathrm{~d} z^{2}}=\frac{F(L-z)}{Y I}
$$

where $Y$ is the elastic modulus of the belt and $I$ is its momentum of inertia. The local strain in the belt is $\epsilon=y / R$, and the corresponding electric field along $z$ axis is (Figure 3C)

$$
E_{z}=\frac{\epsilon}{d}=\frac{y}{d R}
$$

This is the electric field that dominates the potential distribution under small bending approximation and the ignorance of the electric field effect on local strain via the piezoelectric effect. For simplicity of analytical calculation to illustrate the physical principle, we consider the potential at the two side surfaces $y= \pm T / 2$ by integrating the electric field along the entire length of the belt

$V^{ \pm}=\int E \mathrm{~d} s= \pm \int \frac{T}{2 d} \frac{1}{R} \mathrm{~d} s= \pm \frac{T}{2 d} \int \mathrm{d} \theta= \pm \frac{a}{d} \theta_{\text {max }}$

where $\theta_{\max }$ is the maximum deflection angle at the top of the wire. By solving eq 1 and using eq 2, we have

$$
V^{ \pm}= \pm \frac{T F L^{2}}{4 d Y I}
$$

Using the relationship between the maximum deflection $y_{\mathrm{m}}$ and the applied force

$$
F=\frac{3 Y I y_{m}}{L^{3}}
$$

we have the potential induced by the piezoelectric effect at the stretched and compressed side surfaces, respectively, being

$$
V^{ \pm}= \pm 3 T y_{m} / 4 L d
$$

For the purpose of proposing the mechanism for the piezoelectric generator, we now examine the electric contact in the measurement system we have designed. For the wire/ belt used in the measurements, the contacts at the top and the root are nonsymmetric. At the top of the wire, since the work function of $\mathrm{Pt}$ is $6.1 \mathrm{eV}$ and the electron affinity of $\mathrm{ZnO}$ is $4.5 \mathrm{eV},{ }^{14}$ the $\mathrm{Pt}-\mathrm{ZnO}$ contact is Schottky. ${ }^{15}$ The contact at the bottom is $\mathrm{ZnO}$ with silver paste. Since the 


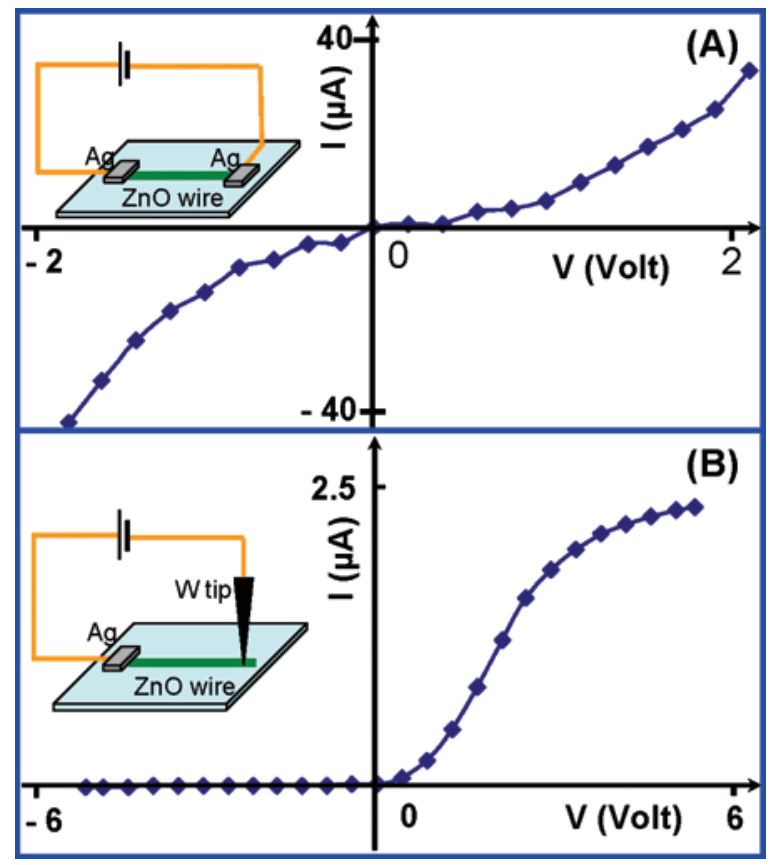

Figure 4. Experiments to verify that the contact between silver paste with $\mathrm{ZnO}$ wire/belt is Ohmic. (A) $I-V$ characteristic of a $\mathrm{ZnO}$ wire with both ends in contact with silver paste. (B) $I-V$ characteristic of a $\mathrm{ZnO}$ wire with one end in contact with silver paste and the other end with tungsten tip.

electron affinity of $\mathrm{ZnO}$ is $4.5 \mathrm{eV}^{14}$ and the work function of $\mathrm{Ag}$ is $4.26 \mathrm{eV},{ }^{16}$ there is no barrier at the interface; thus, the contact between $\mathrm{ZnO}$ and $\mathrm{Ag}$ is likely to be Ohmic. To verify this expected result, we have done the following experiment. If we use the silver paste to connect both ends of a wire, the current that flowed through the system was rather high and the IV curve shows symmetry, indicating that the entire system has the Ohmic contact characteristic (Figure 4A). This is possible only if the contact between $\mathrm{Ag}$ and $\mathrm{ZnO}$ is Ohmic. Alternatively, if one end is in contact a tungsten tip and the other end is with $\mathrm{Ag}$, the $I-V$ characteristic of the system is Schottky (Figure 4B). This is apparent because the $\mathrm{W}-\mathrm{ZnO}$ contact is Schottky. Therefore, the transport property of the experimental setup as shown in Figures 1 and 2 is governed by the Schottky contact at the interface between the AFM Pt tip and $\mathrm{ZnO}$ wire/belt.

We now examine the contact between the AFM conductive tip with the stretched and compressed side surfaces of the belt. As discussed above, the compressed side of the semiconductor $\mathrm{ZnO}$ wire/belt has negative potential $V^{-}$and the stretched side has positive potential $\left(V^{+}\right)$. This is the cause of the two distinct transport processes across the Schottky barrier at the interface, as described below. When the tip contacts the stretched side surface, the Pt metal tip has a potential of nearly zero, $V_{\mathrm{m}}=0$, and the metal tip-belt interface is negatively biased for $\Delta V=V_{\mathrm{m}}-V^{+}<0$. With consideration the n-type semiconductor characteristic of the as-synthesized $\mathrm{ZnO}$ belt, the $\mathrm{Pt}$ metal- $\mathrm{ZnO}$ semiconductor $(\mathrm{M}-\mathrm{S})$ interface in this case is a reversely biased Schottky diode (Figure 3E), resulting in little current flowing across the interface. In this case, the piezoelectric static charges, mainly due to $\mathrm{Zn}^{2+}$ and $\mathrm{O}^{2-}$ ions, are accumulated and preserved, but without creating a current flow through the belt. This is a key process that prevents the slow "leakage" of the current as the deformation is being built up by the tip; otherwise, there would be no observable output electric signal in the next step. As the tip continues to scan and touches the compressed side of the belt (Figure 3F), the metal tip-belt interface is positively biased for $\Delta V=V_{\mathrm{L}}=\mathrm{V}_{\mathrm{m}}-$ $V^{-}>0$. The interface is thus a positively biased Schottky diode, and it is possible to have current flow from the tip through the belt. The flow of electrons is to neutralize the piezoelectric ionic charges distributed in volume, resulting in a sudden increase in the output electric current. The output voltage measured on the load is negative in reference to the grounded root of the belt with consideration the flowing direction of the current.

The elastic deformation energy as created by the displacement force $F$ is mainly dissipated in three ways: creating mechanical resonance/vibration after releasing the belt (as schematically shown in Figure 5A), generating piezoelectric discharge energy for each cycle of the vibration, and overcoming the friction/viscosity, if any, from the environment and substrate. The mechanical resonance of the belt continues for many cycles, but it is eventually damped by the viscosity of the medium. The piezoelectric voltage output is generated in each cycle of the vibration, but the AFM tip in the current experimental design may be too slow to collect the electric signal output from each cycle of the belt vibration. In our experiment, we have found that the discharge signal can sometimes be collected for an extensive period of time, during which the belt may have resonated for over 10 cycles, as shown in Figure 5A, producing a continuous and constant output dc voltage. The resonance frequency of the wire was $\sim 10 \mathrm{kHz}$, and the scanning speed of the tip was $\sim 10 \mu \mathrm{m} / \mathrm{s}$; it is very feasible that the wire had contacted the AFM over 100 times before it departed too far to be in touch. The corresponding optical image of the belt shows a blurred trace, which is an indication of many cycles of vibration of the belt. This observation unambiguously shows that the piezoelectric output voltage is created in each cycle of the vibration. If we can find a way to continuously collect the output voltage, we simply could have a dc power source.

As illustrated in Figure 3D, there is current flow only when the AFM tip is in contact with the compressed side of the belt/wire. If the AFM tip contacts the stretched side, no output current is possible even under extremely large elastic deformation. This expected result is observed experimentally as shown in Figure $5 \mathrm{~B}$, where a $\mathrm{ZnO}$ wire is subjected to a large deformation, but no output voltage is received.

As presented for most of the experimental observations, the output voltage is always negative in reference to the grounded root of the wire/belt. We did, however, have a couple of occasions where a positive output voltage was observed at the very beginning when the tip contacted the wire from above (Figure 5C), but it quickly disappeared for the subsequent scans, and the later measurements showed 


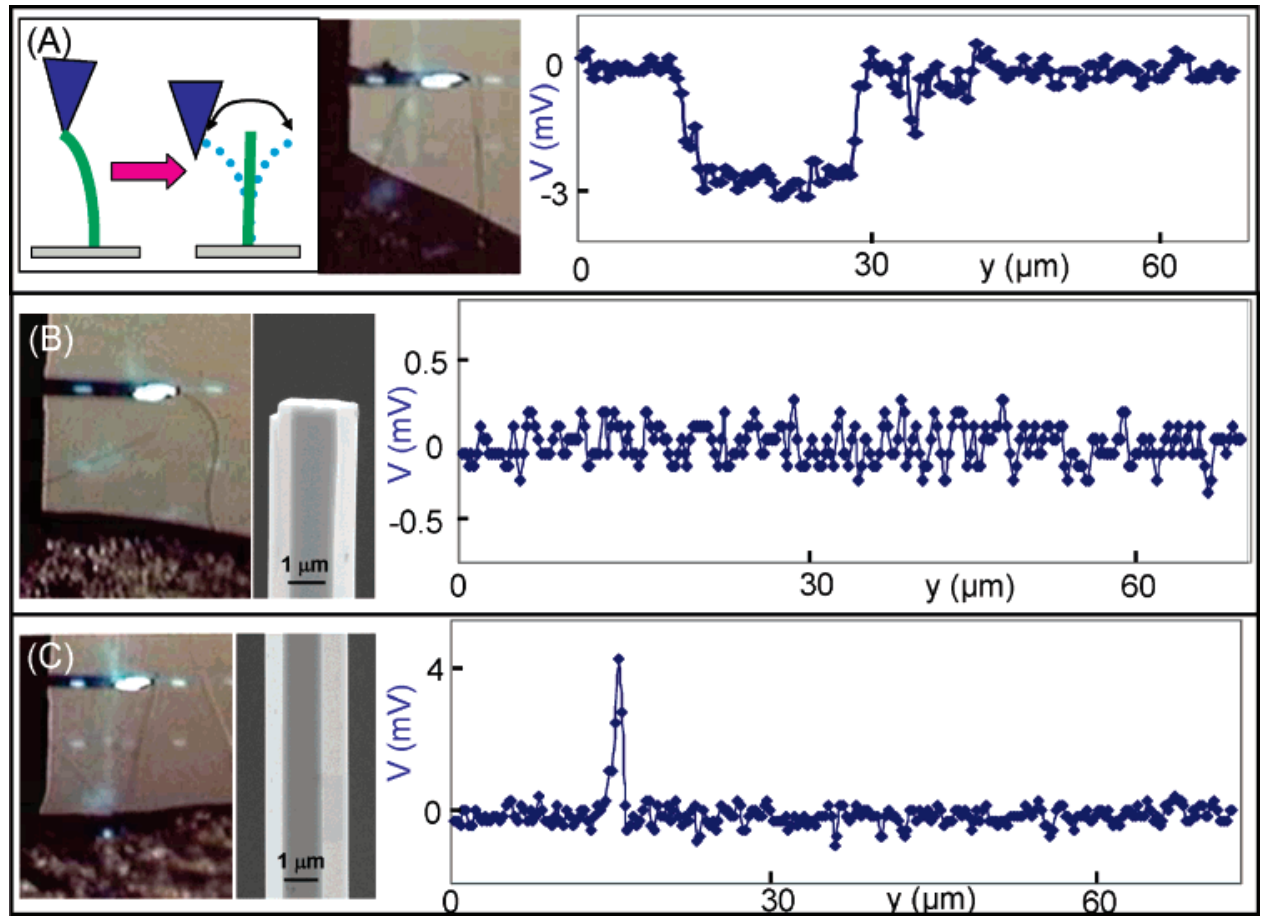

Figure 5. Three occasionally observed phenomena in the AFM manipulation of a $\mathrm{ZnO}$ wire/wire, showing some unique characteristics for the power generator that may be missed in most of cases due to the slow response of the AFM tip in scanning speed and data output rates. (A) Schematic of the resonance vibration of a wire after being released by the AFM tip, showing that the stored elastic energy is transferred mainly into vibration energy after creating the piezoelectric discharge event. But piezoelectric power is generated in each cycle of the vibration. The output voltage image in the right-hand side with a flat and wide plateau is a result of continuous collection of the output voltage from the over 10 cycles of the wire vibration. (B) A largely bent wire and its corresponding SEM image, but with no output voltage signal. (C) A bent wire, its corresponding SEM image, and a positive output voltage peak at the very beginning of the AFM deformation, possibly corresponding to a breakdown of the Schottky barrier at the tip-wire interface due to a larger local potential. This phenomenon was rarely observed.

only negative output voltage peaks. We suspect that the positive voltage peak may be produced by the breakdown of the Schottky barrier under high local piezoelectric voltage when the tip contacted the stretched side of the wire. It must be pointed out that such a phenomenon was observed only a couple of times among the over 20 experiments we have done.

In summary, this paper presents the experimental observation of piezoelectric output of a single $\mathrm{ZnO}$ wire/belt and illustrates the fundamental process of converting mechanical energy into electricity on a nanoscale. By deflecting a wire/ belt using a conductive AFM tip in contact mode, the energy is first created by the deflection force and stored by piezoelectric potential and later converted into piezoelectric energy. By simultaneously acquiring the topography and voltage output images, the piezoelectric discharge process has been investigated as a nanoscale power generator. The mechanism of the generator is the result of coupled semiconducting and piezoelectric properties of $\mathrm{ZnO}$. The piezoelectric effect is required to create electric potential of ionic charges from elastic deformation; semiconducting property is required to preserve the charges and then release the potential via the rectifying behavior of the Schottky barrier at the metal- $\mathrm{ZnO}$ interface, which serves as a switch in the entire process. The good conductivity of $\mathrm{ZnO}$ is rather unique here and it makes the current flow possible. This process may also be possible for wurtzite structured materials such as $\mathrm{GaN}$ and $\mathrm{ZnS}$. But if we replaced $\mathrm{ZnO}$ by $\mathrm{PZT}$, for instance, such a phenomenon may not be observed because PTZ is a dielectric that prevents the current flow through its volume.

The principle and technology demonstrated here have the potential of converting mechanical movement energy (such as body movement, muscle stretching, blood pressure), vibration energy (such as acoustic/ultrasonic wave), and hydraulic energy (such as flow of body fluid, blood flow, contraction of blood vessels, dynamic fluid in nature) into electric energy that may be sufficient for self-powering nanodevices and nanosystems. ${ }^{17}$ The technology could have important applications in wireless self-powered nanodevices by harvesting energy from the environment. It also provides a method for indirectly charging a battery. It is also possible to fabricate a large-power output electric generator by using arrays of $\mathrm{ZnO}$ wires/belts, which can be grown on substrates such as metal foils, flexible organic plastic substrates, ceramic substrates (such as alumina), and compound semiconductors (such as $\mathrm{GaN}$ and $\mathrm{AlN}$ ). The nanogenerator could be the basis for exploring new self-powering technology for in situ, real-time, and implantable biosensing, biomedical monitoring, and biodetection. It could have the potential of solving the key energy requirement for remote sensing and actuating. The methodology and applications demonstrated in this paper simply open a new field in nanotechnology. 
Acknowledgment. Thanks to support from NSF, the NASA Vehicle Systems Program and Department of Defense Research and Engineering (DDR\&E), and the Defense Advanced Research Projects Agency. Thanks to Dr. Xudong Wang for his kind help in the electrical measurements.

Supporting Information Available: Two videos of the experimental process and output images. This material is available free of charge via the Internet at http://pubs.acs.org.

\section{References}

(1) Pan, Z. W.; Dai, Z. R.; Wang, Z. L. Science 2001, 291 (5510), 19471949.

(2) Gao, P. X.; Ding, Y.; Mai, W. J.; Hughes, W. L.; Lao, C. S.; Wang, Z. L. Science 2005, 309 (5741), 1700-1704.

(3) Wang, X. D.; Summers, C. J.; Wang, Z. L. Nano Lett. 2004, 4 (3), 423-426.

(4) Gao, P. X.; Wang, Z. L. Appl. Phys. Lett. 2004, 84 (15), 28832885.

(5) Look, D. C.; Coskun, C.; Claflin, B.; Farlow, G. C. Physica B 2003, $340,32-38$.
(6) Verghese, P. M.; Clarke, D. R. J. Appl. Phys. 2000, 87 (9), 44304438

(7) Zhao, M. H.; Wang, Z. L.; Mao, S. X. Nano Lett. 2004, 4 (4), 587590.

(8) Wang, Z. L.; Song, J. H. Science 2006, 312, 242-246.

(9) Kim, S.; Kang, B. S.; Ren, F.; Ip, K.; Heo, Y. W.; Norton, D. P.; Pearton, S. J. Appl. Phys. Lett. 2004, 84 (10), 1698-1700.

(10) Duan, X. F.; Huang, Y.; Agarwal, R.; Lieber, C. M. Nature 2003, 421 (6920), 241-245.

(11) Zheng, G. F.; Patolsky, F.; Cui, Y.; Wang, W. U.; Lieber, C. M. Nat. Biotechnol. 2005, 23 (10), 1294-1301.

(12) Bai, X. D.; Gao, P. X.; Wang, Z. L.; Wang, E. G. Appl. Phys. Lett. 2003, 82, 4806.

(13) Song, J. H.; Wang, X. D.; Riedo, E.; Wang, Z. L. Nano Lett. 2005 5 (10), 1954-1958.

(14) Hasegawa, S.; Nishida, S.; Yamashita, T.; Asahi, H. J. Ceram Process. Res. 2005, 6, 245.

(15) Park, W. I.; Yi, G. C.; Kim, J. W.; Park, S. M. Appl. Phys. Lett 2003, 82 (24), 4358-4360.

(16) Yannouleas, C.; Landman, U. Phys. Rev. B 2000, 61 (23), 1589515904.

(17) US patent pending.

NL060820V 